\title{
The miR-136-5p/ROCK1 axis suppresses invasion and migration, and enhances cisplatin sensitivity in head and neck cancer cells
}

\author{
BO YANG ${ }^{1}$, JIAN ZANG $^{1}$, WEILI YUAN ${ }^{2}$, XUEJUN JIANG ${ }^{1}$ and FANG ZHANG $^{3}$ \\ ${ }^{1}$ Department of Otorhinolaryngology Head and Neck Surgery, The First Hospital of China Medical University, \\ Shenyang, Liaoning 110001; Departments of ${ }^{2}$ Oral and Maxillofacial Surgery and ${ }^{3}$ Otorhinolaryngology Head and \\ Neck Surgery, The Fourth Affiliated Hospital of China Medical University, Shenyang, Liaoning 110032, P.R. China
}

Received March 4, 2020; Accepted December 15, 2020

DOI: $10.3892 /$ etm.2021.9748

\begin{abstract}
Laryngeal squamous cell carcinoma (LSCC) and hypopharyngeal squamous cell carcinoma (HPSCC) are two types of head and neck cancers with high incidence rates and relatively poor prognoses. The aim of the present study was to determine the effects of microRNA (miR/miRNA)-136-5p and its downstream target, Rho-associated coiled-coil containing protein kinase 1 (ROCK1), on LSCC and HPSCC progression and cisplatin sensitivity. The miRNA and protein expression levels in head and neck cancer cell lines were evaluated using reverse transcription-quantitative PCR and western blotting, respectively. MTT, wound healing assays, transwell assays and flow cytometry analysis were performed to measure cell properties. The binding between miR-136-5p and ROCK1 was detected using a dual-luciferase reporter assay. Autophagy double-labeled adenoviral infection assays were used to assess cell autophagy. The results showed that miR-136-5p was expressed in LSCC and HPSCC cells. Functional experiments showed that the expression of miR-136-5p in LSCC and HPSCC cells was negatively correlated with cell viability, invasion and migration. Additionally, miR-136-5p overexpression inhibited epithelial-mesenchymal transition, whereas miR-136-5p knockdown had the opposite effect. Dual-luciferase reporter assays confirmed the targeting relationship between miR-136-5p and ROCK1. miR-136-5p overexpression increased the cisplatin sensitivity of LSCC and HPSCC cells by reducing cell viability, as well as promoting cell apoptosis and autophagy. miR-136-5p overexpression decreased the expression levels of its downstream target
\end{abstract}

Correspondence to: Dr Fang Zhang, Department of Otorhinolaryngology Head and Neck Surgery, The Fourth Affiliated Hospital of China Medical University, 4 East Chongshan Road, Shenyang, Liaoning 110032, P.R. China

E-mail: zhangfang_cmu4h@163.com

Key words: cell invasion, cell migration, cisplatin sensitivity, laryngeal squamous cell carcinoma, hypopharyngeal squamous cell carcinoma, microRNA-136-5p, Rho-associated coiled-coil containing protein kinase 1
ROCK1 and attenuated activity of the Akt/mTOR signaling pathway in cisplatin-treated LSCC and HPSCC cells. Conversely, miR-136-5p knockdown increased ROCK1 levels and decreased cisplatin sensitivity of the LSCC and HPSCC cells by increasing cell viability and inhibiting cell apoptosis, which was reversed by ROCK1 inhibition using the ROCK1 inhibitor, Y27632. Taken together, the results showed that the miR-136-5p/ROCK1 axis inhibits cell invasion and migration, and increases the sensitivity of LSCC and HPSCC cells to cisplatin.

\section{Introduction}

Head and neck cancer is one of the most common types of cancer worldwide which includes the mouth, pharynx (nasopharynx, oropharynx and hypopharynx), larynx and paranasal sinus. According to the biological characteristics of head and neck cancer, it can be divided into several types. The most common type is head and neck squamous cell carcinoma (HNSCC), which accounts for $>95 \%$ of all cases of head and neck cancer (1). HNSCC is the sixth most common type of cancer worldwide, with 600,000 cases diagnosed each year and a mortality rate of $40-50 \%(2,3)$. The majority of patients with locally advanced HNSCC usually develop a locoregional recurrence and/or distant metastases, and only a few patients with a locoregional recurrence may recover following treatment surgery and/or re-irradiation (3). Overall, the 5-year survival rate for patients with HNSCC has remained at 40-60\% and is accompanied by a relatively poor prognosis $(1,4,5)$. Laryngeal squamous cell carcinoma (LSCC) and hypopharyngeal squamous cell carcinoma (HPSCC) are two types of HNSCC with high incidence rates and a relatively poor prognosis following treatment (6-8). Although multiple proteins and pathways have been shown to be associated with the development of LSCC and HPSCC, the exact molecular mechanisms of action and potential therapeutic targets affecting proliferation and migration remain to be elucidated (9).

MicroRNAs (miRNAs/miRs) are noncoding small RNA molecules that regulate the expression of target genes by binding to the $3^{\prime}$-untranslated region (UTR). miRNAs are involved in various biological processes including cell proliferation, apoptosis, invasion and migration (10). Various 
miRNAs exhibit altered expression levels in cancers and serve crucial roles in the development of several types of cancer (11). Previous studies have found that the expression levels of miR-136 are significantly decreased at the tumor site and that miR-136 acts as a tumor suppressor in various tumor types, participating in the development of tumors by regulating the expression levels of downstream apoptosis-related genes $(12,13)$. Additionally, miR-136 has also been found to be associated with cisplatin resistance in human epithelial ovarian and gastric cancers $(14,15)$. Therefore, miR-136 may be a potential target for cancer therapy.

A previous study reported the relationship between miR-136 and Rho-associated coiled-coil containing protein kinase (ROCK) $1(16,17)$. ROCK1, one of the isoforms of the ROCK, is a downstream effector of Rho $\mathrm{A}$ and is activated when it selectively binds to GTP $(18,19)$. Activated ROCK interacts with the actin cytoskeleton to promote the formation of stress fibers and focal adhesions, which in turn promote the metastatic ability of tumor cells (20-22). ROCK overexpression has been reported to be associated with the progression of various malignancies, including bladder cancer, liver cancer and breast cancer $(23,24)$. ROCK downregulation inhibits tumor growth and metastasis, and enhances the efficacy of cisplatin (25-27). In addition, the epithelial-mesenchymal transition (EMT) is a key process which often precedes and facilitates local invasion, vascular migration and distant metastasis of tumors (28). A previous study has shown that the ROCK pathway is not only involved in the proliferation, migration, adhesion and morphological changes of various cell types, but also in the EMT of tumor cells (22). ROCK serves a key role in TGF- $\beta$-induced EMT, which promotes mesenchymal transformation by rapidly activating RhoA-dependent signaling pathways (29). Therefore, ROCK serves a crucial role in cancer development.

Based on the aforementioned points, the regulatory mechanism of action behind the miR-136/ROCK1 axis in LSCC and HPSCC were investigated in the present study and its role in cell invasion and migration, as well as cisplatin sensitivity, were assessed.

\section{Materials and methods}

Cell cultures, transfection and treatment. FaDu cells were purchased from Procell and cultured in an incubator at $37^{\circ} \mathrm{C}$ and $5 \% \mathrm{CO}_{2}$ in minimum essential media (MEM, Sigma-Aldrich; Merck KGaA) containing 10\% FBS (Hyclone, GE Healthcare Life Sciences). FD-LSC-1 cells were obtained from Fudan University, China, and cultured in a BEBM (Lonza Group, Ltd.) supplemented with $10 \%$ FBS and 1\% L-glutamine at $37^{\circ} \mathrm{C}$ with $5 \% \mathrm{CO}_{2}$. To overexpress miR-136-5p, miR-136-5p mimics or mimics-negative controls (NC) were transfected into $\mathrm{FaDu}$ or FD-LSC-1 cells for $24 \mathrm{~h}$ at $37^{\circ} \mathrm{C}$. To downregulate miR-136-5p expression, $\mathrm{FaDu}$ or FD-LSC-1 cells were transfected with miR-136-5p inhibitor or inhibitor-NC for $24 \mathrm{~h}$ at $37^{\circ} \mathrm{C}$. The transfections were performed using Lipofectamine ${ }^{\circledR} 2000$ (Invitrogen; Thermo Fisher Scientific, Inc.). For the cisplatin+miR-136-5p mimics/inhibitor/mimics $\mathrm{NC} /$ inhibitor NC group and cisplatin+miR-136-5p inhibitor+Y2763 group, after transfection with the miR-136-5p mimics, inhibitor or NC for $24 \mathrm{~h}, \mathrm{FaDu}$ and FD-LSC-1 cells were immediately treated with cisplatin (2.6 $\mu \mathrm{M}$, Sigma-Aldrich; Merck KGaA) and/or Y2763 (25 $\mu \mathrm{M}$, MedChem Express, Ltd.) for $24 \mathrm{~h}$. After transfection and treatment, the cells were taken immediately for further experiments. The sequences for the miRNA mimics and inhibitors were as follows: hsa-miR-136-5p mimics, 5'-ACUCCAUUUGUUU UGAUGAUGGA-3'; 5'-CAUCAUCAAAACAAAUGGAGU UU-3'; NC mimics, 5'-UUCUCCGAACGUGUCACGUTT-3'; 5'-ACGUGACACGUUCGGAGAATT-3'; hsa-miR-136-5p inhibitor, 5'-UCCAUCAUCAAAACAAAUGGAGU-3'; NC inhibitor, 5'-UUGUACUACACAAAAGUACUG-3'. The sequences were purchased from Jintuosi Biological Technology Co., Ltd.

Reverse transcription-quantitative PCR (RT-qPCR). Total RNA was extracted from cells using a Total RNA extraction kit (Tiangen Biotech, Co., Ltd.). cDNA was synthesized using an RNase inhibitor (Tiangen Biotech, Co., Ltd.), Super M-MLV reverse transcriptase (BioTeke Corporation), 5x PCR buffer and dNTP (2.5 mM each). The miR-136-5p primer used for reverse transcription was: 5'-GTTGGCTCTGGTGCAGGGTC CGAGGTATTCGCACCAGAGCCAACTCCATC-3'. The temperature protocol that was used for reverse transcription was: $37^{\circ} \mathrm{C}$ for $30 \mathrm{~min}, 42^{\circ} \mathrm{C}$ for $30 \mathrm{~min}$ and $70^{\circ} \mathrm{C}$ for $15 \mathrm{~min}$. To detect the expression levels of miRNA-136-5p, qPCR was performed using PCR Master Mix (Tiangen Biotech, Co., Ltd.), SYBR-Green (Beijing Solarbio Science \& Technology Co., Ltd.) and specific primers for the target genes. The thermocycling conditions used for qPCR were as follows: $94^{\circ} \mathrm{C}$ for $4 \mathrm{~min}, 40$ cycles of $94^{\circ} \mathrm{C}$ for $15 \mathrm{sec}, 60^{\circ} \mathrm{C}$ for $20 \mathrm{sec}$ and $72^{\circ} \mathrm{C}$ for $15 \mathrm{sec}$. The $2^{-\Delta \Delta \mathrm{Cq}}$ comparative method was used for data analysis (30). 5S ribosomal RNA (rRNA) was used as the internal control to normalize the expression levels of genes. The value of each control was adjusted to 1 in each individual repeat of the RT-qPCR assay, therefore the mean value of the control is always 1 . The primers were synthesized by GenScript. The primer sequences were as follows: hsa-miR-136-5p forward, 5'-ACTCCATTTGTTTTGATGATGGA-3' and reverse, 5'-GCAGGGTCCGAGGTATTC-3'; and 5S rRNA forward, 5'-GATCTCGGAAGCTAAGCAGG-3' and reverse, 5'-TGGTGCAGGGTCCGAGGTAT-3'.

Cell viability assay. MTT assays were performed to determine the cell viability. Briefly, cells were seeded in 96-well plates at a density of $4 \times 10^{3}$ cells per well. Subsequently, MTT $(0.5 \mathrm{mg} / \mathrm{ml})$ was added to each well. After incubating for $4.5 \mathrm{~h}$ at $37^{\circ} \mathrm{C}$ in an incubator with $5 \% \mathrm{CO}_{2}$, the supernatant was removed and $150 \mu 1 \mathrm{DMSO}$ was added to each well to dissolve the purple crystals. Cells were immersed in the dark for $10 \mathrm{~min}$ and the optical density values at $570 \mathrm{~nm}$ were measured using a Microplate Reader (Biotek Instruments, Inc.).

Cell migration assay. Wound healing assays were performed to assess cell migration. The cells of each group were cultured to the fusion state ( $90 \%$ confluence). After transfection for $24 \mathrm{~h}$ as aforementioned, FaDu cells were cultured in a minimum essential media without serum and treated with $1 \mu \mathrm{g} / \mathrm{ml}$ mitomycin C (Sigma-Aldrich; Merck KGaA) for $1 \mathrm{~h}$. FD-LSC-1 cells were cultured in a BEBM without serum and treated with $1 \mu \mathrm{g} / \mathrm{ml}$ mitomycin $\mathrm{C}$ for $1 \mathrm{~h}$. Subsequently, the 
cells in each group were scratched with a $200 \mu$ l pipette tip and washed with serum-free medium to remove cell debris. After scratching, serum-free medium was used for cell culture and pictures were taken under a light microscope (magnification, $\mathrm{x} 100$; Olympus Corporation) to assess wound closure at 0 and $24 \mathrm{~h}$.

Invasion assay. Cell invasion was detected using a Transwell assay. Briefly, Transwell chambers (Corning, Inc.) pre-coated with Matrigel were placed in 24-well plates. Culture medium (MEM medium for FaDu cells and BEBM medium for FD-LSC-1 cells, $800 \mu 1$ ) supplemented with $30 \%$ FBS was added to the lower chamber. Cell suspension in $200 \mu \mathrm{l}$ serum-free media was added to the upper chamber at a density of $1.5 \times 10^{4}$ cells/well. After incubation in a cell culture incubator at $37^{\circ} \mathrm{C}$ with $5 \% \mathrm{CO}_{2}$ for $24 \mathrm{~h}$, the transwell chambers were washed three times with PBS to remove non-invading cells. The cells on the lower chamber were fixed with $4 \%$ paraformaldehyde at room temperature for $25 \mathrm{~min}$ and then stained with $0.4 \%$ crystal violet solution for $5 \mathrm{~min}$ at room temperature. Cells on lower chamber were counted under an inverted light microscope (magnification, $\mathrm{x} 200$; Olympus Corporation). A total of five fields were selected for each sample and the mean cell numbers was presented.

Western blotting analysis. The protein expression levels were detected using western blotting. Briefly, total protein was extracted from cells using RIPA lysis buffer (Beijing Solarbio Science \& Technology Co., Ltd.) and phenylmethanesulfonyl fluoride protease inhibitor (Beijing Solarbio Science \& Technology Co., Ltd.). Protein concentration in the lysate was quantified using a BCA protein concentration assay kit (Beijing Solarbio Science \& Technology Co., Ltd.). Subsequently, $20 \mu \mathrm{g}$ protein was loaded onto a $10 \%$ SDS-gel and resolved using SDS-PAGE. After the protein was transferred to the PVDF membranes (EMD Millipore), the membranes were incubated with primary antibodies at $4^{\circ} \mathrm{C}$ overnight. The primary antibodies used in the present study were: E-cadherin (cat. no. 20874-1-AP, RRID:AB_10697811; 1:10,000; ProteinTech Group, Inc.), N-cadherin (cat. no. 22018-1-AP, RRID:AB_2813891; 1:5,000; ProteinTech Group, Inc.), vimentin (cat. no. 10366-1-AP, RRID:AB_2273020; 1:5,000; ProteinTech Group, Inc.), ROCK1 (cat. no. 21850-1-AP, RRID:AB_10953526; 1:1,000; ProteinTech Group, Inc.), Akt (cat. no. AF6259, RRID: AB_2835120; 1:1,000; Affinity Biosciences), phospho-(p-)-Akt (Ser473; cat. no. AF0016, RRID: AB_2810275; 1:1,000; Affinity Biosciences), glycogen synthase kinase-3 $\beta$ (GSK-3 $\beta$ ) (cat. no. AF5016; RRID: AB_2834935; 1:1,000; Affinity Biosciences), p-GSK-3 $\beta$ (Ser9; cat.no. AF2016, RRID: AB_2834439; 1:1,000; Affinity Biosciences), mTOR (cat. no. AF7803, RRID: AB_2844167; 1:1,000; Affinity Biosciences), p-mTOR (S2448; cat. no. AF3308, RRID: AB_2834727; 1:1,000; Affinity Biosciences), microtubule-associated protein 1 light chain 3 II/I (LC3II/I; cat. no. A7198; 1:1,000; ABclonal Biotech Co., Ltd.), P62 (cat. no. 18420-1-AP, RRID: AB_10694431; 1:2,000; ProteinTech Group, Inc.), cleaved caspase-3 (cat. no. AF7022, RRID: AB_2835326; 1:1,000; Affinity Biosciences), Bax (cat. no. 50599-2-lg, RRID: AB_2061561; 1:5,000; ProteinTech Group, Inc.), Bcl-2 (cat. no. 12789-1-AP;
RRID: AB_2227948; 1:2,000; ProteinTech Group, Inc.) and $\beta$-actin (cat.no. sc-47778; RRID: AB_2714189; 1:1,000; Santa Cruz Biotechnology, Inc.). After incubating with horseradish peroxidase-conjugated goat anti-rabbit/mouse secondary antibodies (1:5,000; cat. nos. A0208 and A0216; Beyotime Institute of Biotechnology) for $45 \mathrm{~min}$ at $37^{\circ} \mathrm{C}$, signals were visualized using enhanced chemiluminescence solution (Beyotime Institute of Biotechnology). The expression levels of protein were normalized to $\beta$-actin. The value of each control was adjusted to 1 in each individual repeat of the western blot, therefore the mean value of the control is always 1 .

Dual-luciferase reporter assay. The binding site of miR-136-5p and ROCK1 was predicted using TargetScanHuman 7.2 (http://www.targetscan.org/vert_72/). miR-136-5p was searched and the multiple genes that miR-136-5p may target were obtained. UTRs were also searched between ROCK 1 and miR-136-5p to obtain the targeted binding sequence between miR-136-5p and ROCK1.

For the verification of the relationship between miR-136-5p and ROCK1, the wild-type (wt) ROCK1-3'-UTR sequence and the mutant (mut) ROCK1-3'-UTR sequence were cloned into the pmirGLO dual-luciferase reporter vectors (Genscript Biotech Corporation) to construct pmirGLO-ROCK1-3'-UTR-wt and pmirGLO-ROCK1-3'-UTR-mut plasmids. 293T cells were harvested and seeded in 12-well plates. Subsequently, the plasmids were co-transfected with miR-136-5p mimics/mimic-NC or miR-136-5p inhibitor/inhibitor-NC into $293 \mathrm{~T}$ cells using Lipofectamine ${ }^{\circledR} 2000$ reagent. After 48 h of incubation, the transfected cells were immediately harvested and assayed for luciferase activity using the dual-luciferase reporter assay system (Promega Corporation) according to the manufacturer's protocol. Firefly luciferase activity was normalized to Renilla luciferase activity.

Apoptosis assay. Apoptosis was detected using flow cytometry analysis. The Annexin V- FITC apoptosis detection kit (Beyotime Institute of Biotechnology) was used to detect apoptosis. Cells were harvested and adjusted to a density of $1 \times 10^{6}$ cells/tube. Subsequently, cells were treated with $5 \mu 1$ Annexin V-FITC and $10 \mu \mathrm{l}$ PI and incubated for $15 \mathrm{~min}$ at room temperature in the dark. Finally, cells stained with Annexin V-FITC and PI were detected using flow cytometry (ACEA Bioscience, Inc.) and analyzed using NovoExpress 1.2.5 (ACEA Biosciences, Inc.).

Autophagy double-labeled adenovirus infection assay. Cells were seeded in a 24 -well plate $\left(5 \times 10^{4}\right.$ cells) and infected with RFP-GFP-LC3-labeled adenovirus (Hanbio Biotechnology Co., Ltd.) at a multiplicity of infection of 50. After incubating for $24 \mathrm{~h}$ in an incubator at $37^{\circ} \mathrm{C}$ supplied with $5 \% \mathrm{CO}_{2}$, the supernatant of the medium containing the virus solution was discarded and replaced with complete medium (MEM medium containing 10\% FBS for FaDu cells and BEBM medium containing $10 \%$ FBS for FD-LSC-1 cells). After incubating for $24 \mathrm{~h}$ in an incubator at $37^{\circ} \mathrm{C}$, images were taken using a laser-scanning confocal microscope equipped with the FV10-ASW system (magnification, x400; Olympus Corporation). 
A

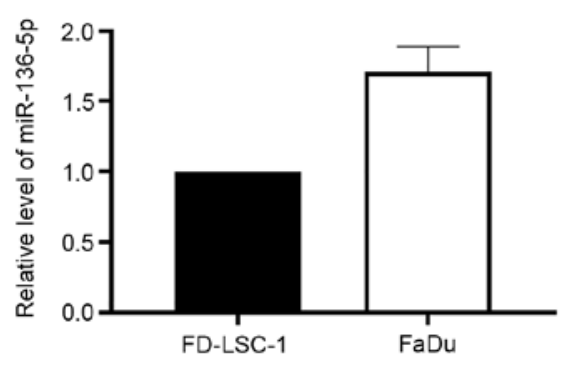

B

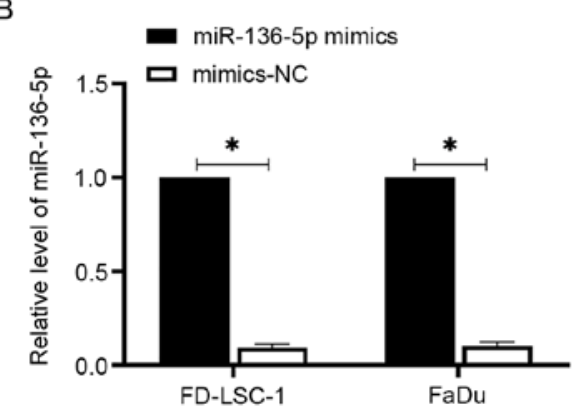

C

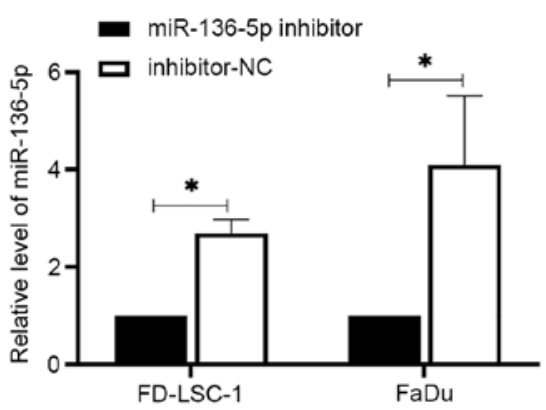

Figure 1. The expression levels of miR-136-5p in LSCC and HPSCC cells. (A) The expression level of miR-136-5p in LSCC and HPSCC cells. Following transfection of the cells with (B) miR-136-5p mimics, mimics-NC; or (C) miR-136-5p inhibitor or inhibitor-NC for $24 \mathrm{~h}$, the expression levels of miR-136-5p were measured using reverse transcription-quantitative PCR. Data are presented as the mean $\pm \mathrm{SD}, \mathrm{n}=3$. ${ }^{*} \mathrm{P}<0.05$. HPSCC, head and neck squamous cell carcinoma; LSCC, laryngeal squamous cell carcinoma; miR, microRNA; NC, negative control.

Statistical analysis. Unpaired t-tests were used to analyze differences between two groups. Comparisons among multiple groups were analyzed using a one-way ANOVA with Tukey's multiple comparisons test. The experiments were performed at least three times. Data are presented as the mean \pm SD and analyzed using GraphPad version 8.0. $\mathrm{P}<0.05$ was considered to indicate a statistically significant difference.

\section{Results}

miR-136-5p suppresses the viability, invasion and migration of LSCC and HPSCC cells. To detect the expression levels of miR-136-5p in LSCC and HPSCC cells, RT-qPCR was performed. The results showed that miR-136-5p was expressed in FD-LSC-1 and FaDu cells (Fig. 1A). The effects of miR-136-5p on LSCC and HPSCC cell functions were further investigated. Cells were transfected with miR-136-5p mimics, mimics-NC, miR-136-5p inhibitor or inhibitor-NC for 24 h. miR-136-5p mimics increased the expression levels of miR-136-5p in FD-LSC-1 and FaDu cells, whereas miR-36-5p inhibitor decreased miR-136-5p levels (Fig. 1B and C, respectively; $\mathrm{P}<0.05)$. MTT assays were subsequently performed to detect the viability of the transfected cells. The results showed that miR-136-5p overexpression reduced the cell viability of FD-LSC-1 and FaDu cells, whereas miR-136-5p downregulation promoted cell viability compared with the respective $\mathrm{NC}$ transfected cells (Fig. 2A, $\mathrm{P}<0.05$ ). Wound healing and transwell invasion assays were used to measure cell migration and invasion, respectively. The results showed that miRNA-136-5p overexpression reduced cell invasion and migration, whereas miRNA-136-5p knockdown increased the migratory and invasive capacity of FD-LSC-1 and FaDu cells compared with the corresponding NC groups (Fig. 2B-F; all $\mathrm{P}<0.05)$. Western blotting was performed to measure E-cadherin, $\mathrm{N}$-cadherin and vimentin protein expression levels in the transfected cells. As shown in Fig. 2G, miR-136-5p overexpression increased the protein expression of E-cadherin but decreased the $\mathrm{N}$-cadherin and vimentin levels compared with the mimics-NC group in both cell lines (all $\mathrm{P}<0.05$ ). Similarly, miRNA-136-5p inhibition decreased E-cadherin levels and increased $\mathrm{N}$-cadherin and vimentin levels compared with the inhibitor-NC group $(\mathrm{P}<0.05)$. These results suggested that miR-136-5p reduces cell viability, invasion and migration in LSCC and HPSCC cells.

miRNA-136-5p directly targets ROCK1 in LSCC and HPSCC cells. ROCK1 was identified as a potential target of miR-136-5p, which was confirmed by the luciferase reporter assays. A ROCK1-wt luciferase plasmid containing the potential miR-136-5p binding sites as well as a mut version (ROCK1-mut) was generated (Fig. 3A). As shown in Fig. 3B, the luciferase activity in LSCC and HPSCC cells transfected with ROCK1-wt plasmid was significantly reduced by the transfection of miR-136-5p mimics $(\mathrm{P}<0.05)$, whereas no alteration was observed in the luciferase activity of cells transfected with ROCK1-mut plasmid ( $P>0.05)$, which suggested that miR-136-5p may bind to the 3'UTR of ROCK1.

miR-136-5p overexpression increases the cisplatin sensitivity of LSCC and HPSCC cells. Cisplatin is commonly used as a chemotherapeutic drug for several types of cancer. To explore the role of miR-136-5p on the sensitivity of LSCC and HPSCC cells to cisplatin, cells were transfected with miR-136-5p mimics or mimics-NC for $24 \mathrm{~h}$ and then treated with cisplatin $(2.6 \mu \mathrm{M})$ for $24 \mathrm{~h}$. The results of cell viability analysis using 

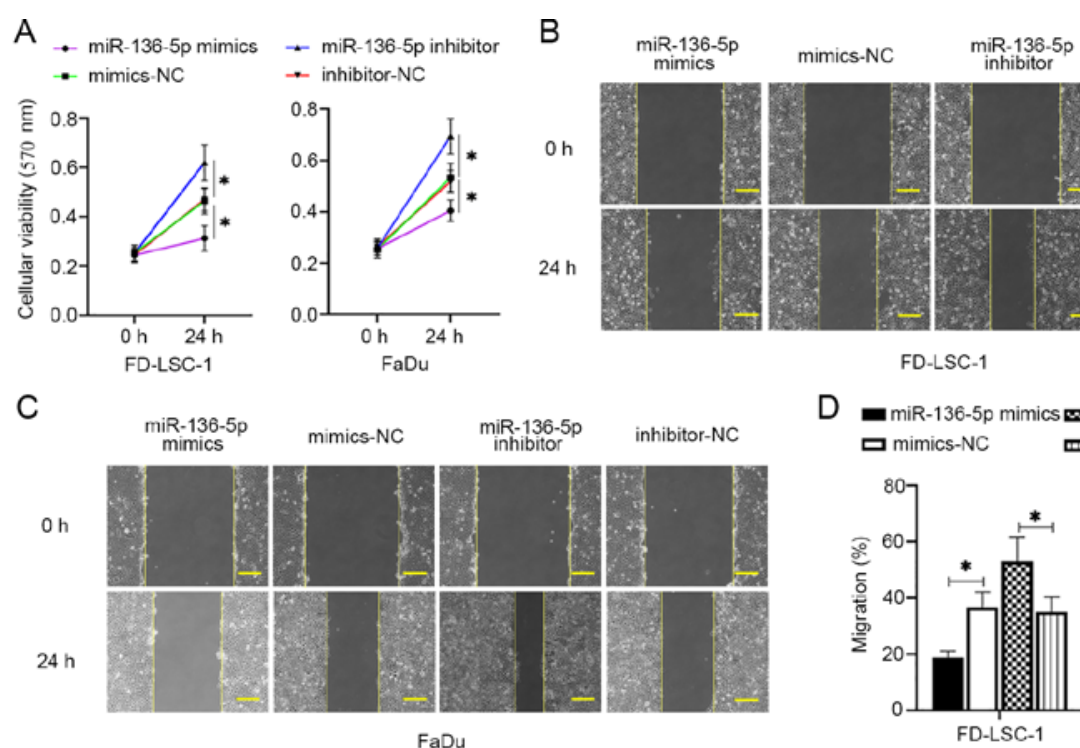

FD-LSC-1

E

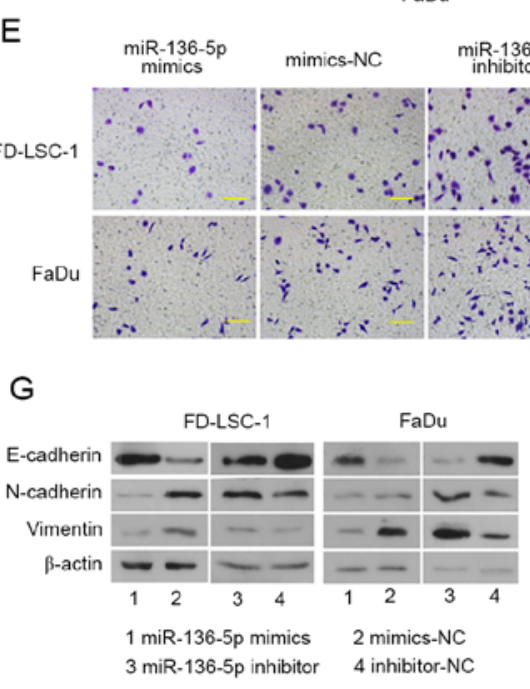

inhibitor-NC

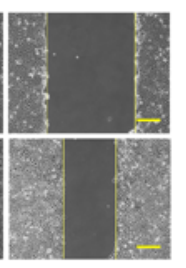

D



$\mathrm{F}$

-miR-136-5p mimics $\mathbf{m i R}$-136-5p inhibitor 口 mimics-NC inhibitor-NC



FD-LSC-1

ma miR-136-5p inhibitor mo inhibitor-NC

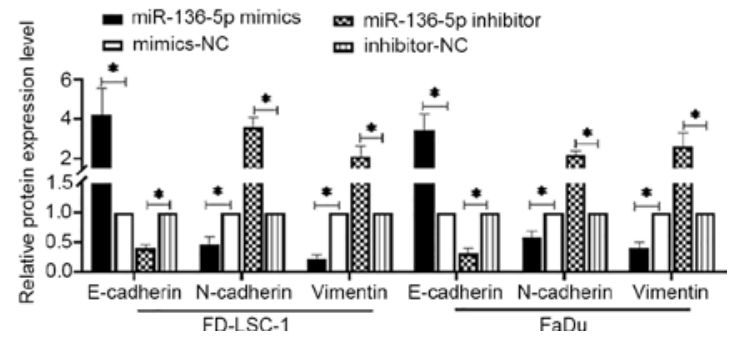

Figure 2. miR-136-5p suppresses viability, invasion and migration of LSCC and HPSCC cells. (A) After LSCC and HPSCC cells transfected with miR-136-5p mimics, mimics-NC, miR-136-5p inhibitor or inhibitor-NC for $24 \mathrm{~h}$, cell viability was detected using MTT assays, $\mathrm{n}=6$. Cell migratory abilities were measured using wound healing assays in (B) FD-LSC-1 cells and (C) FaDu cells, scale bar=200 $\mu \mathrm{m}$. (D) Migratory rates were then quantified. (E) Cell invasion was observed and $(\mathrm{F})$ measured using transwell assays, scale bar $=100 \mu \mathrm{m}$. (G) The protein expression levels of E-cadherin, N-cadherin and vimentin were measured using western blot analysis and normalized to the levels of $\beta$-actin. Data are presented as the mean $\pm \mathrm{SD}, \mathrm{n}=3$. ${ }^{*} \mathrm{P}<0.05$. HPSCC, head and neck squamous cell carcinoma; LSCC, laryngeal squamous cell carcinoma; miR, microRNA; NC, negative control.

MTT assays showed a decrease in the cell viability in response to cisplatin treatment following miR-136-5p overexpression (Fig. 4A; $\mathrm{P}<0.05$ ). Apoptosis analysis measured by flow cytometry showed that miR-136-5p overexpression combined with cisplatin significantly promoted apoptosis (Fig. 4B and C; $\mathrm{P}<0.05)$. The effect of cisplatin coupled with miR-136-5p on autophagy was also assessed. The results showed that miR-136-5p upregulation resulted in the increase of free yellow puncta (indicating autophagosome) and red puncta (indicating autolysosomes), indicating that autophagosome formation was promoted and the autophagy flux was induced by miR-136-5p upregulation (Fig. 4D and 4E). As shown in Fig. 4F, miR-136-5p overexpression significantly decreased the protein expression levels of ROCK1 and P62, as well as the ratios of $\mathrm{p}$-Akt, $\mathrm{p}-\mathrm{GSK}-3 \beta$ and $\mathrm{p}$-mTOR to their unphosphorylated versions, but increased LC3II/I levels in LSCC and HPSCC cells (all $\mathrm{P}<0.05$ ).
miR-136-5p downregulation decreases the cisplatin sensitivity of LSCC and HPSCC cells by targeting ROCK1. The effects of the miR-136-5p/ROCK axis on the sensitivity of LSCC and HPSCC cells to cisplatin were investigated. Cells were transfected with miR-136-5p inhibitor or inhibitor-NC for $24 \mathrm{~h}$ and then treated with cisplatin $(2.6 \mu \mathrm{M})$ and/or Y27632 $(25 \mu \mathrm{M}$, ROCK inhibitor) for $24 \mathrm{~h}$. As shown in Fig. 5A-C, miR-136-5p downregulation promoted cisplatin-treated cell viability and inhibited apoptosis whereas ROCK1 knockdown reversed the effects of miR-136-5p downregulation on cisplatin-treated cell viability and apoptosis (all $\mathrm{P}<0.05$ ). Additionally, cleaved caspase-3, Bax and Bcl-2 are important regulators of apoptosis. Cleaved caspase-3 and Bax promote apoptosis whereas Bcl-2 inhibits apoptosis $(31,32)$. The results of western blot analysis showed that miR-136-5p downregulation increased the protein expression levels of ROCK1, p-GSK-3 $\beta$ and Bcl-2, but decreased cleaved caspase- 3 and Bax levels, whereas 


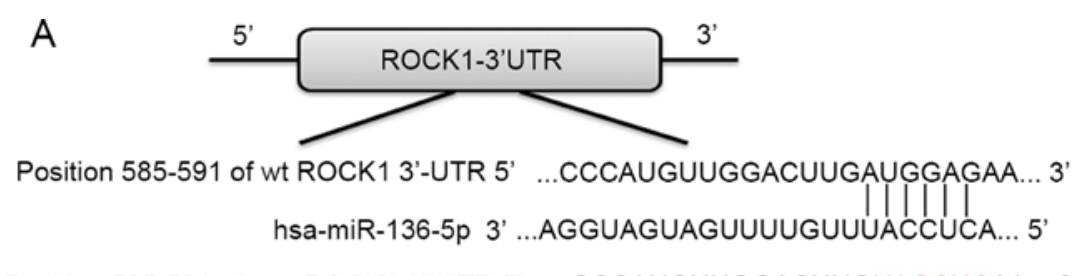

Position 585-591 of mut ROCK1 3'-UTR 5' ...CCCAUGUUGGACUUGUACCUCAA... 3



Figure 3. miRNA-136-5p directly targets ROCK1 in LSCC and HPSCC cells. (A) Binding sites between ROCK1 wt and the mut-type miR-136-5p are shown. (B) miR-136-5p mimics or mimic-NC and luciferase plasmid containing ROCK1-wt or ROCK1-mut transcript were co-transfected into LSCC and HPSCC cells, luciferase activity was measured using dual-luciferase reporter assays. Data are presented as the mean $\pm \mathrm{SD}, \mathrm{n}=3$. ${ }^{*} \mathrm{P}<0.05$. HPSCC, head and neck squamous cell carcinoma; LSCC, laryngeal squamous cell carcinoma; miR, microRNA; NC, negative control; UTR, untranslated region; wt, wild-type.

ROCK1 downregulation reversed the effects of miR-136-5p knockdown on the protein expression levels (Fig. 5D; all $\mathrm{P}<0.05)$.

\section{Discussion}

The role of miRNAs in cancer has been extensively investigated, particularly their downstream targets, biological functions and significance in cancer development and progression (11). miR-136 has been reported to be involved in the development of several types of cancer through targeting downstream effector molecules and is associated with cisplatin resistance in cancer $(14,33)$. Based on the important roles of miR-136-5p in cancer development, the potential regulatory mechanisms of action of miR-136-5p in LSCC and HPSCC progression, as well as cisplatin sensitivity were investigated. In the present study, it was shown that miR-136-5p inhibited cell invasion and migration, and increased the sensitivity of LSCC and HPSCC to cisplatin by repressing ROCK1 expression.

miR-136 is a negative regulator of cancer progression and its expression levels are reduced during the development of various types of cancer, including gastric cancer, colon cancer and breast cancer (33-35). Functional experiments indicate that miR-136 can inhibit cell proliferation, invasion and metastasis and induce apoptosis during cancer progression $(15,35,36)$. In particular, it has been reported that downregulation of miR-136-5p markedly reduces cell viability, invasion and migration in LSCC (37). Consistent with the previous results, the present study identified the suppressive functions of miR-136-5p on cell viability, invasion and migration during
LSCC and HPSCC progression. The EMT is an important process that regulates cancer development, initiation and metastatic dissemination (38). A significant feature of EMT is the reduction in epithelial markers, usually indicated by the presence of E-cadherin and the elevation of mesenchymal markers, such as $\mathrm{N}$-cadherin and vimentin, resulting in an invasive phenotype (39). Yan et al (35) investigated the effects of miR-136 on the EMT process in breast cancer. The results showed that miR-136 overexpression significantly increases E-cadherin levels but decreases vimentin levels, suggesting that miR-136 suppresses EMT. In the present study, it was shown that miR-136-5p overexpression significantly increased E-cadherin expression levels and decreased $\mathrm{N}$-cadherin and vimentin levels, whereas miR-136-5p downregulation reversed these changes. This suggested that miR-135-5p suppresses the EMT process in LSCC and HPSCC cells. Phenotypic changes between epithelial and mesenchymal are widely recognized as important factors influencing tumor cell invasion and metastasis (40). Thus, miR-136-5p may inhibit cell migration and invasion through mechanisms dependent on EMT (41). Additionally, a previous study found that $\mathrm{Bcl}-2$ is a downstream target of miR-136-5p and that miR-136-5p promotes apoptosis by promoting the degradation of the apoptosis inhibitor, Bcl-2, which is negatively associated with cancer development (42). Collectively, the results indicated the tumor-suppressive function of miR-136-5p on LSCC and HPSCC development.

TargetScan 7.2 predicted that miR-576-3p targeted ROCK1. It has been reported that ROCK1 is a downstream target of miR-136-5p and that the miR-136-5p/ROCK1 axis participates in the inflammatory response induced by cerebral 
A

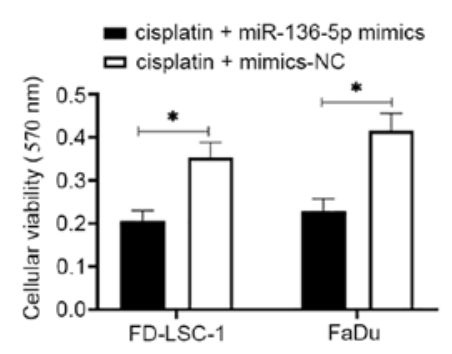

D

D



B

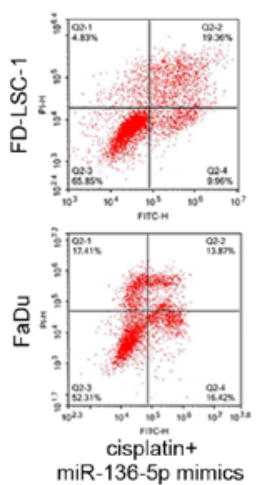

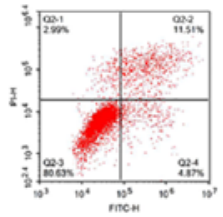

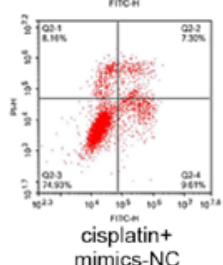

C

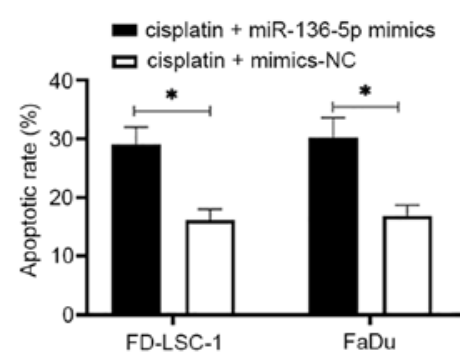

E

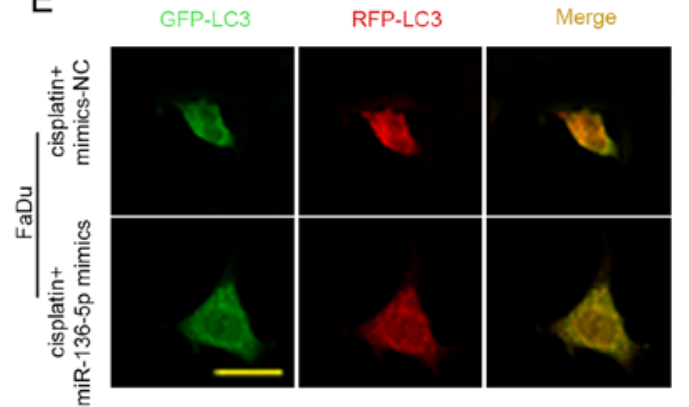

F
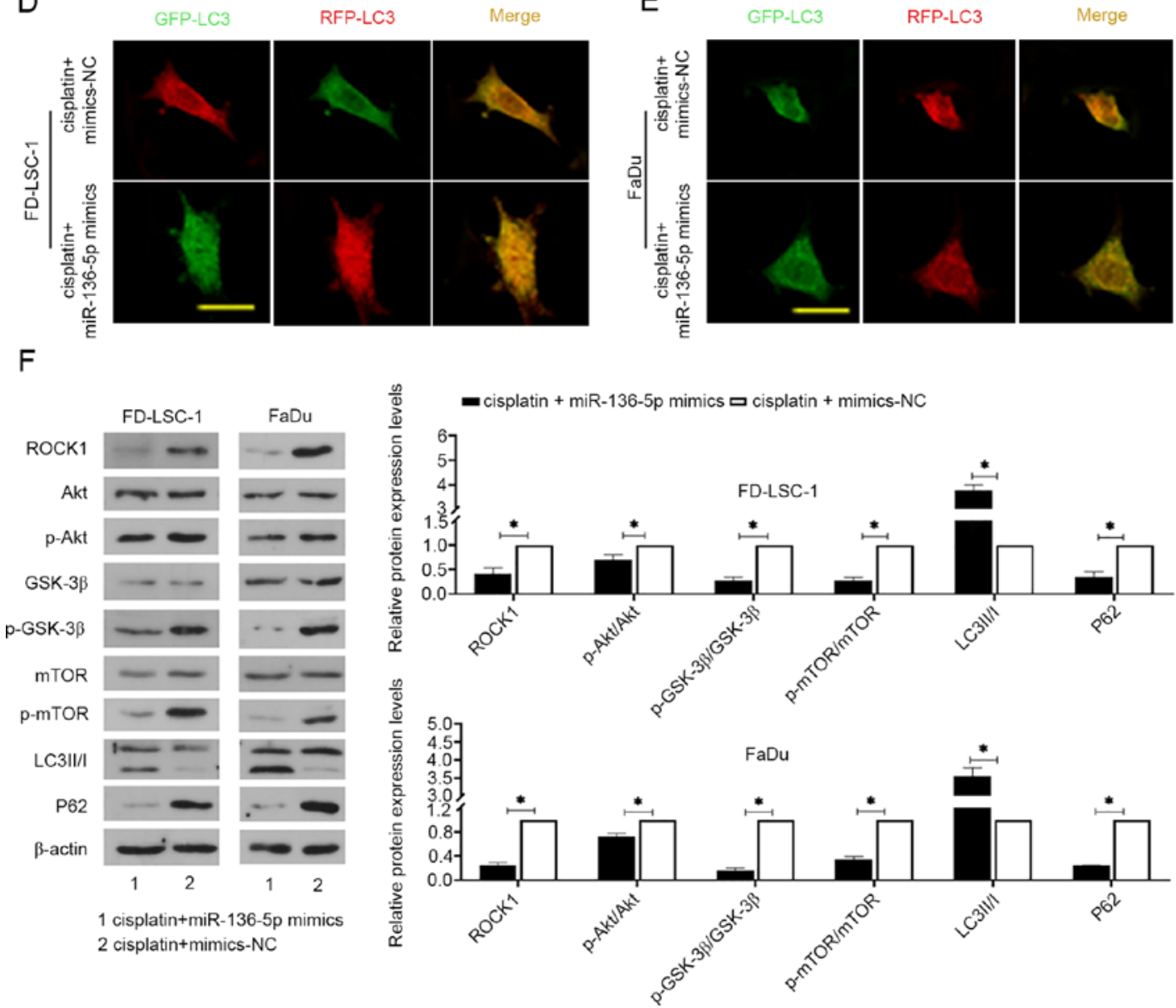

Figure 4. miR-136-5p overexpression increases the cisplatin sensitivity of laryngeal squamous cell carcinoma and head and neck squamous cell carcinoma cells. (A) After cells were transfected with miR-136-5p mimics or mimics-NC and treated with cisplatin (2.6 $\mu \mathrm{M})$ for $24 \mathrm{~h}$, cell viability was detected using MTT assays, $n=6$. (B) Flow cytometric detection of apoptosis using annexin V-FITC/PI staining and (C) quantification of apoptosis. Autophagy was detected using an autophagy double-labeled adenovirus infection assay in (D) FD-LSC-1 and (E) FaDu cells, scale bar=50 $\mu$ m. Free yellow puncta indicates the autophagosome, free red puncta indicates the autophagolysosome. (F) The protein expression levels of ROCK1, p-Akt/Akt (Ser473), GSK-3 $\beta$, p-GSK-3 $\beta$ (Ser9), p-mTOR (S2448)/mTOR, LC3II/I and P62 were measured using western blot analysis and normalized to the levels of $\beta$-actin. Data are presented as the mean $\pm \mathrm{SD}, \mathrm{n}=3$. * $\mathrm{P}<0.05$. GSK-3 $\beta$, glycogen synthase kinase-3 $\beta$; LC3, microtubule-associated protein 1 light chain 3 ; miR, microRNA; mTOR, mammalian target of rapamycin; NC, negative control; p-, phosphorylated.

ischemia/reperfusion injury, as well as in the proliferation and invasion of LSCC cells $(16,17)$. The present study also confirmed this relationship using a dual-luciferase reporter assay. ROCK1 is a serine-threonine protein kinase, which phosphorylates a variety of downstream target proteins through interactions with Rho GTPases, to promote the generation of actin-myosin mediated contractile forces, thereby controlling cell mobility, metastasis and invasion $(22,43)$. Previous studies have indicated that ROCK1 is associated with cancer progression and its expression levels are elevated in several types of cancer (44-46). For example, in prostate cancer, ROCK expression levels have been reported to be significantly increased and this was associated with increased cell proliferation and migration (47). Additionally, it has been reported that ROCK1 expression is positively correlated with tumor size and lymph node metastasis, and that downregulation of ROCK1 inhibits cell proliferation, migration and invasion of LSCC cells $(48,49)$. The present study demonstrated that miR-136-5p 
A cisplatin + miR-136-5p inhibitor
口 cisplatin + miR-136-5p inhibitor + Y27632 cisplatin + inhibitor-NC

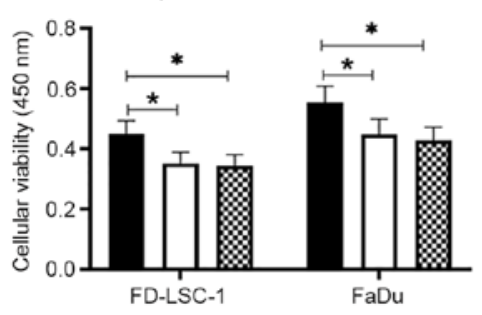

C
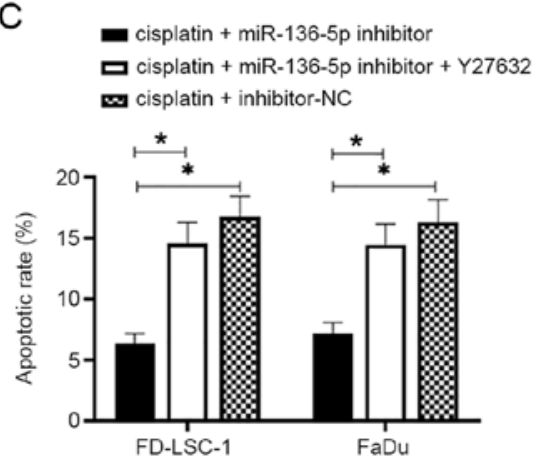

D

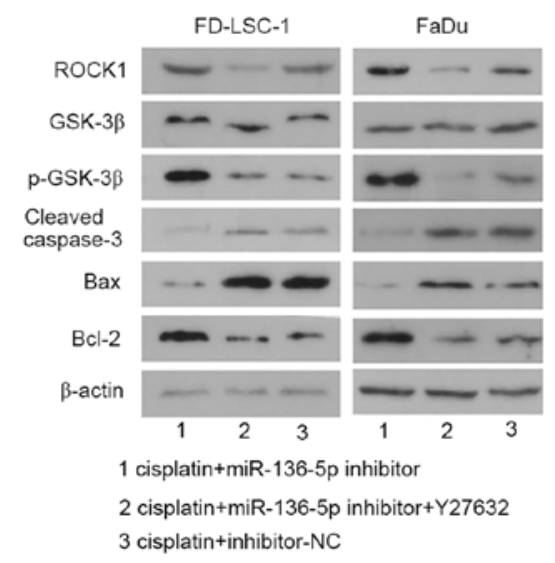

B
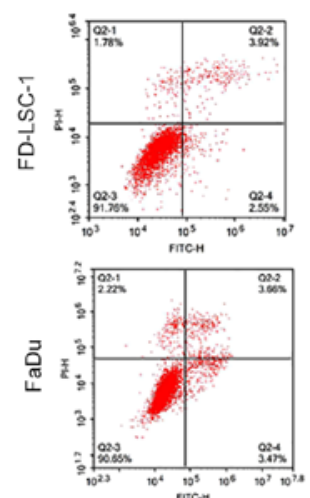

cisplatin+

miR-136-5p inhibitor
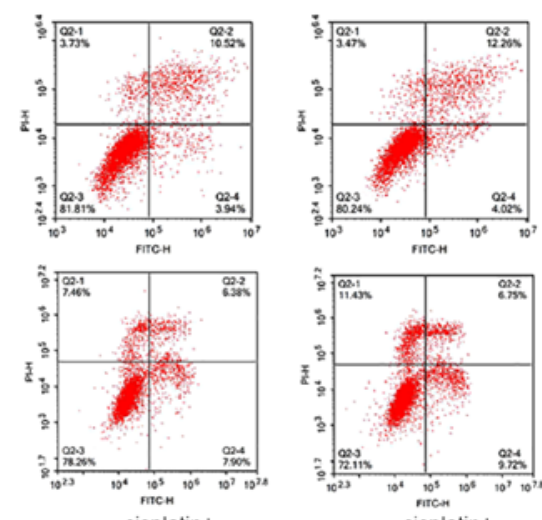

miR-136-5p inhibitor+Y27632

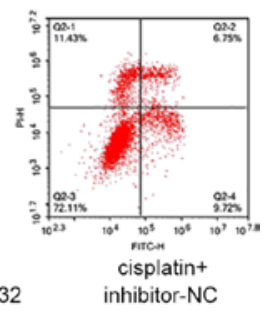

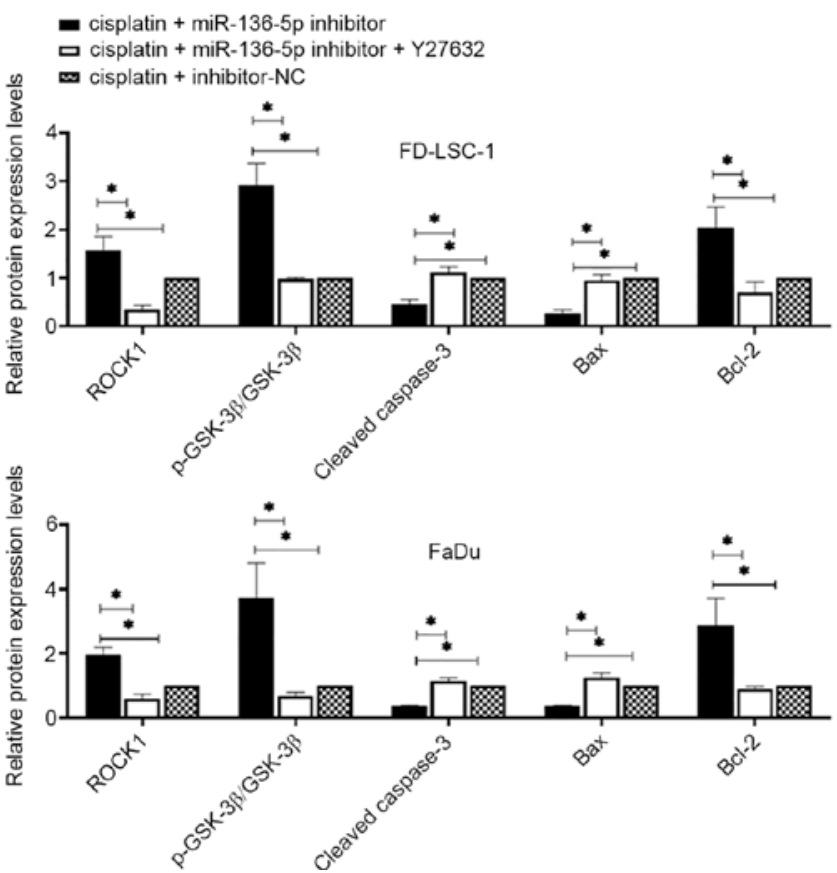

Figure 5. miR-136-5p downregulation decreased the cisplatin sensitivity of laryngeal squamous cell carcinoma and head and neck squamous cell carcinoma cells by targeting ROCK1. (A) After cells were transfected with miR-136-5p inhibitor or inhibitor-NC for $24 \mathrm{~h}$, as well as with cisplatin (2.6 $\mu \mathrm{M})$ and/or Y27632 $(25 \mu \mathrm{M}$, ROCK inhibitor) for $24 \mathrm{~h}$, cell viability was detected using MTT assays, $\mathrm{n}=6$. (B) Flow cytometric detection of apoptosis using annexin V-FITC/PI staining and (C) quantification of apoptosis. (D) The protein expression levels of ROCK1, p-GSK-3 $\beta$ (Ser9)/GSK-3 $\beta$, cleaved caspase-3, Bax and Bcl-2 were measured using western blot analysis and normalized to the levels of $\beta$-actin (D). Data are presented as the mean $\pm \mathrm{SD}, \mathrm{n}=3$. ${ }^{*} \mathrm{P}<0.05$. GSK-3 $\beta$, glycogen synthase kinase-3 $\beta$; miR, microRNA; NC, negative control; p-, phosphorylated; ROCK, Rho-associated coiled-coilcontaining protein kinase.

may also regulate cell metastasis and invasion by regulating the downstream target ROCK1 in LSCC and HPSCC cells.

Cisplatin is a systemic therapy, usually combined with radiation as a standard treatment of head and neck cancer (50). Cisplatin-based chemotherapy regimens are the most commonly used adjunctive therapy for several types of cancer (51). However, cisplatin resistance is a major barrier to therapeutic success (52). Thus, increasing the chemosensitivity to cisplatin may contribute to the successful treatment for various types of cancer. Zhao et al (14) demonstrated that the expression of miR-136 was associated with primary cisplatin resistance in human epithelial ovarian cancer. Studies have reported that miR-136 reversed cisplatin chemosensitivity in glioma cells through targeting downstream targets (53). The present study showed that miR-136-5p overexpression increased the sensitivity to cisplatin of LSCC and HPSCC cells by reducing cell viability and promoting apoptosis. Autophagy is a complex process that often occurs in normal cells and is used to degrade excess protein and subcellular components in lysosomes (54). Under normal conditions, autophagy regulates the metabolism of proteins, maintains homeostasis and inhibits tumor formation (55). The p-Akt/ 
p-mTOR axis negatively regulates autophagy (56). GSK-3 $\beta$ is a major downstream molecule of p-Akt that inhibits autophagy by activating mTOR (57). LC3-I is cleaved and lipidated to form LC3-II during autophagosome formation and LC3-II is a known autophagosomal marker in mammals (58). P62 is a selective autophagy substrate that can be continuously degraded by autophagy (59). Mathew et al (60) demonstrated that autophagy inhibited tumorigenesis by interfering with the p62 pathway, which is crucial for tumorigenesis. Wu et al (61) reported that autophagy decreases the sensitivity of lung adenocarcinomas to cisplatin treatment through the activation of the AMPK/mTOR signaling pathway. The present study showed that miR-136-5p overexpression combined with cisplatin decreased P62 levels and inhibited the Akt/mTOR pathway, a pathway that negatively regulates autophagy (62). The results suggested that miR-136-5p overexpression promoted autophagy, which is conducive to the inhibition of LSCC and HPSCC development, and the increase of cisplatin sensitivity of LSCC and HPSCC cells. Conversely, miR-136-5p knockdown decreased the sensitivity to cisplatin in both LSCC and HPSCC cells, while the inhibition of ROCK1 reversed the effects of miR-136-5p knockdown on cisplatin sensitivity, indicating that miR-136-5p may affect the cisplatin sensitivity of LSCC and HPSCC cells by targeting ROCK1. Collectively, the present study suggested that miR-136-5p renders LSCC and HPSCC cells more sensitive to cisplatin treatment and miR-136-5p and cisplatin combined promotes chemosensitivity through targeting ROCK1 in LSCC and HPSCC cells. Therefore, the miRNA-136-5p/ROCK axis may serve as a promising therapeutic target for the treatment of LSCC and HPSCC.

\section{Acknowledgements}

Not applicable.

\section{Funding}

No funding was received.

\section{Availability of data and materials statements}

The datasets used and/or analyzed during the current study are available from the corresponding author on reasonable request.

\section{Authors' contributions}

BY and FZ designed the study and wrote the manuscript. JZ and WY performed the data collection and confirmed the authenticity of all the raw data. XJ performed statistical analysis. All authors read and approved the final manuscript.

\section{Ethics approval and consent to participate}

Not applicable.

\section{Patient consent for publication}

Not applicable.

\section{Competing interests}

The authors declare that they have no competing interests.

\section{References}

1. Chan JYK, Zhen G and Agrawal N: The role of tumor DNA as a diagnostic tool for head and neck squamous cell carcinoma. Semin Cancer Biol 55: 1-7, 2019.

2. Torre LA, Bray F, Siegel RL, Ferlay J, Lortet-Tieulent J and Jemal A: Global cancer statistics, 2012. CA Cancer J Clin 65: 87-108, 2015.

3. Solomon B, Young RJ and Rischin D: Head and neck squamous cell carcinoma: Genomics and emerging biomarkers for immunomodulatory cancer treatments. Semin Cancer Biol 52: 228-240, 2018.

4. Grégoire V, Lefebvre JL, Licitra L and Felip E; EHNS-ESMO-ESTRO Guidelines Working Group: Squamous cell carcinoma of the head and neck: EHNS-ESMO-ESTRO Clinical Practice Guidelines for diagnosis, treatment and follow-up. Ann Oncol 21 (Suppl 5): v184-v186, 2010.

5. Ferlay J, Soerjomataram I, Dikshit R, Eser S, Mathers C, Rebelo M, Parkin DM, Forman D and Bray F: Cancer incidence and mortality worldwide: Sources, methods and major patterns in GLOBOCAN 2012. Int J Cancer 136: E359-E386, 2015.

6. Ferlay J, Shin HR, Bray F, Forman D, Mathers C and Parkin DM: Estimates of worldwide burden of cancer in 2008: GLOBOCAN 2008. Int J Cancer 127: 2893-2917, 2010.

7. Fong PY, Tan SH, Lim DWT, Tan EH, Ng QS, Sommat K, Tan DSW and Ang MK: Association of clinical factors with survival outcomes in laryngeal squamous cell carcinoma (LSCC). PLoS One 14: e0224665, 2019.

8. Cristina V, Herrera-Gómez RG, Szturz P, Espeli V and Siano M: Immunotherapies and future combination strategies for head and neck squamous cell carcinoma. Int J Mol Sci 20: 20, 2019.

9. Nowicka Z, Stawiski K, Tomasik B and Fendler W: Extracellular miRNAs as Biomarkers of Head and Neck Cancer Progression and Metastasis. Int J Mol Sci 20: 20, 2019.

10. Bushati N and Cohen SM: microRNA functions. Annu Rev Cell Dev Biol 23: 175-205, 2007.

11. Lee YS and Dutta A: MicroRNAs in cancer. Annu Rev Pathol 4: 199-227, 2009

12. Libbus BL and Johnson LA: The creeping vole, Microtus oregoni: Karyotype and sex-chromosome differences between two geographical populations. Cytogenet Cell Genet 47: 181-184, 1988 .

13. Zhang Y, Li Y, Han L, Zhang P and Sun S: SUMO1P3 is associated clinical progression and facilitates cell migration and invasion through regulating miR-136 in non-small cell lung cancer. Biomed Pharmacother 113: 108686, 2019.

14. Zhao H, Liu S, Wang G, Wu X, Ding Y, Guo G, Jiang J and Cui S: Expression of miR-136 is associated with the primary cisplatin resistance of human epithelial ovarian cancer. Oncol Rep 33: 591-598, 2015.

15. Yu L, Zhou GQ and Li DC: miR-136 triggers apoptosis in human gastric cancer cells by targeting AEG-1 and BCL2. Eur Rev Med Pharmacol Sci 22: 7251-7256, 2018.

16. Zhong Y, Yu C and Qin W: lncRNA SNHG14 promotes inflammatory response induced by cerebral ischemia/reperfusion injury through regulating miR-136-5p /ROCK1. Cancer Gene Ther 26: 234-247, 2019.

17. Zhang W, Shi J, Cheng C and Wang H: CircTIMELESS regulates the proliferation and invasion of lung squamous cell carcinoma cells via the miR-136-5p/ROCK1 axis. J Cell Physiol 235: 5962-5971, 2020.

18. Riento K and Ridley AJ: Rocks: Multifunctional kinases in cell behaviour. Nat Rev Mol Cell Biol 4: 446-456, 2003.

19. Ishizaki T, Maekawa M, Fujisawa K, Okawa K, Iwamatsu A, Fujita A, Watanabe N, Saito Y, Kakizuka A, Morii N, et al: The small GTP-binding protein Rho binds to and activates a 160 $\mathrm{kDa}$ Ser/Thr protein kinase homologous to myotonic dystrophy kinase. EMBO J 15: 1885-1893, 1996.

20. Ishizaki T, Naito M, Fujisawa K, Maekawa M, Watanabe $N$, Saito Y and Narumiya S: p160ROCK, a Rho-associated coiled-coil forming protein kinase, works downstream of Rho and induces focal adhesions. FEBS Lett 404: 118-124, 1997.

21. del Peso L, Hernández-Alcoceba R, Embade N, Carnero A, Esteve P, Paje C and Lacal JC: Rho proteins induce metastatic properties in vivo. Oncogene 15: 3047-3057, 1997. 
22. Narumiya S, Tanji $\mathrm{M}$ and Ishizaki T: Rho signaling, ROCK and mDia1, in transformation, metastasis and invasion. Cancer Metastasis Rev 28: 65-76, 2009.

23. Wong CC, Wong CM, Tung EK, Man K and Ng IO: Rho-kinase 2 is frequently overexpressed in hepatocellular carcinoma and involved in tumor invasion. Hepatology 49: 1583-1594, 2009.

24. Morgan-Fisher M, Wewer UM and Yoneda A: Regulation of ROCK activity in cancer. J Histochem Cytochem 61: 185-198, 2013.

25. Bishop AL and Hall A: Rho GTPases and their effector proteins. Biochem J 348: 241-255, 2000

26. Imamura F, Mukai M, Ayaki M and Akedo H: Y-27632, an inhibitor of rho-associated protein kinase, suppresses tumor cell invasion via regulation of focal adhesion and focal adhesion kinase. Jpn J Cancer Res 91: 811-816, 2000.

27. Ohta T, Takahashi T, Shibuya T, Amita M, Henmi N, Takahashi K and Kurachi H: Inhibition of the Rho/ROCK pathway enhances the efficacy of cisplatin through the blockage of hypoxia-inducible factor- $1 \alpha$ in human ovarian cancer cells. Cancer Biol Ther 13: 25-33, 2012.

28. Thiery JP: Epithelial-mesenchymal transitions in tumour progression. Nat Rev Cancer 2: 442-454, 2002.

29. Bhowmick NA, Ghiassi M, Bakin A, Aakre M, Lundquist CA, Engel ME, Arteaga CL and Moses HL: Transforming growth factor-beta1 mediates epithelial to mesenchymal transdifferentiation through a RhoA-dependent mechanism. Mol Biol Cell 12: 27-36, 2001.

30. Livak KJ and Schmittgen TD: Analysis of relative gene expression data using real-time quantitative PCR and the 2(-Delta Delta C(T)) Method. Methods 25: 402-408, 2001.

31. Cohen GM: Caspases: The executioners of apoptosis Biochem J 326: 1-16, 1997.

32. Chipuk JE, Moldoveanu T, Llambi F, Parsons MJ and Green DR: The BCL-2 family reunion. Mol Cell 37: 299-310, 2010.

33. Zheng J, Ge P, Liu X, Wei J, Wu G and Li X: miR-136 inhibits gastric cancer-specific peritoneal metastasis by targeting HOXC10. Tumour Biol 39: 1010428317706207, 2017.

34. Ren H, Qi Y, Yin X and Gao J: miR-136 targets MIEN1 and involves the metastasis of colon cancer by suppressing epithelial-to-mesenchymal transition. OncoTargets Ther 11 : 67-74, 2017.

35. Yan M, Li X, Tong D, Han C, Zhao R, He Y and Jin X: miR-136 suppresses tumor invasion and metastasis by targeting RASAL2 in triple-negative breast cancer. Oncol Rep 36: 65-71, 2016.

36. Yuan Q, Cao G, Li J, Zhang Y and Yang W: MicroRNA-136 inhibits colon cancer cell proliferation and invasion through targeting liver receptor homolog-1/Wnt signaling. Gene 628 : $48-55,2017$.

37. Wang Z, Huang C, Zhang A, Lu C and Liu L: Overexpression of circRNA 100290 promotes the progression of laryngeal squamous cell carcinoma through the miR-136-5p/RAP2C axis. Biomed Pharmacother 125: 109874, 2020.

38. Chaffer CL and Weinberg RA: A perspective on cancer cell metastasis. Science 331: 1559-1564, 2011

39. Cho ES, Kang HE, Kim NH and Yook JI: Therapeutic implications of cancer epithelial-mesenchymal transition (EMT). Arch Pharm Res 42: 14-24, 2019.

40. Chaffer CL, San Juan BP, Lim E and Weinberg RA: EMT, cell plasticity and metastasis. Cancer Metastasis Rev 35: 645-654, 2016.

41. Kang W, Wang Q, Dai Y, Wang H, Wang M, Wang J, Zhang D, Sun P, Qi T, Jin X, et al: Hypomethylation of PlncRNA-1 promoter enhances bladder cancer progression through the miR-136-5p/Smad3 axis. Cell Death Dis 11: 1038, 2020.

42. Young FE: Efficacy of new tests and the safety of the blood supply. Transfusion 30: 4-5, 1990 .

43. Liu S, Goldstein RH, Scepansky EM and Rosenblatt M: Inhibition of rho-associated kinase signaling prevents breast cancer metastasis to human bone. Cancer Res 69: 8742-8751, 2009.

44. Liang H, Zhang C, Guan H, Liu J and Cui Y: lncRNA DANCR promotes cervical cancer progression by upregulating ROCK1 via sponging miR-335-5p. J Cell Physiol 234: 7266-7278, 2019.
45. Du W, Tang H, Lei Z, Zhu J, Zeng Y, Liu Z and Huang JA miR-335-5p inhibits TGF- $\beta 1$-induced epithelial-mesenchymal transition in non-small cell lung cancer via ROCK1. Respir Res 20: 225, 2019

46. Hu C, Zhou H, Liu Y, Huang J, Liu W, Zhang Q, Tang Q, Sheng F, $\mathrm{Li} \mathrm{G}$ and Zhang R: ROCK1 promotes migration and invasion of non small cell lung cancer cells through the PTEN/PI3K/FAK pathway. Int J Oncol 55: 833-844, 2019.

47. Gong H, Zhou L, Khelfat L, Qiu G, Wang Y, Mao K and Chen W: Rho-associated protein kinase (ROCK) promotes proliferation and migration of PC-3 and DU145 prostate cancer cells by targeting LIM kinase 1 (LIMK1) and matrix metalloproteinase-2 (MMP-2). Med Sci Monit 25: 3090-3099, 2019.

48. Zhang J, He X, Ma Y, Liu Y, Shi H, Guo W and Liu L: Overexpression of ROCK1 and ROCK2 inhibits human laryngeal squamous cell carcinoma. Int J Clin Exp Pathol 8: 244-251, 2015

49. Liu Y, Liu J, Wang L, Yang X and Liu X: MicroRNA 195 inhibits cell proliferation, migration and invasion in laryngeal squamous cell carcinoma by targeting ROCK1. Mol Med Rep 16: 7154-7162, 2017

50. Carlsson L, Bratman SV, Siu LL and Spreafico A: The cisplatin total dose and concomitant radiation in locoregionally advanced head and neck cancer: Any recent evidence for dose efficacy? Curr Treat Options Oncol 18: 39, 2017.

51. Dasari S and Tchounwou PB: Cisplatin in cancer therapy: Molecular mechanisms of action. Eur J Pharmacol 740: 364-378, 2014.

52. Amable L: Cisplatin resistance and opportunities for precision medicine. Pharmacol Res 106: 27-36, 2016

53. Chen W, Yang Y, Chen B, Lu P, Zhan L, Yu Q, Cao K and Li Q: miR-136 targets E2F1 to reverse cisplatin chemosensitivity in glioma cells. J Neurooncol 120: 43-53, 2014.

54. Glick D, Barth S and Macleod KF: Autophagy: Cellular and molecular mechanisms. J Pathol 221: 3-12, 2010.

55. Yao Q, Chen J, Lv Y, Wang T, Zhang J, Fan J and Wang L: The significance of expression of autophagy-related gene Beclin, $\mathrm{Bcl}-2$, and Bax in breast cancer tissues. Tumour Biol 32: 1163-1171, 2011

56. Janku F, McConkey DJ, Hong DS and Kurzrock R: Autophagy as a target for anticancer therapy. Nat Rev Clin Oncol 8: 528-539, 2011.

57. Azoulay-Alfaguter I, Elya R, Avrahami L, Katz A and Eldar-Finkelman $\mathrm{H}$ : Combined regulation of mTORC1 and lysosomal acidification by GSK-3 suppresses autophagy and contributes to cancer cell growth. Oncogene 34: 4613-4623, 2015.

58. Tanida I, Ueno T and Kominami E: LC 3 conjugation system in mammalian autophagy. Int J Biochem Cell Biol 36: 2503-2518, 2004

59. Bjørkøy G, Lamark T, Brech A, Outzen H, Perander M, Overvatn A, Stenmark H and Johansen T: p62/SQSTM1 forms protein aggregates degraded by autophagy and has a protective effect on huntingtin-induced cell death. J Cell Biol 171: 603-614, 2005.

60. Mathew R, Karp CM, Beaudoin B, Vuong N, Chen G, Chen HY, Bray K, Reddy A, Bhanot G, Gelinas C, et al: Autophagy suppresses tumorigenesis through elimination of p62. Cell 137: 1062-1075, 2009

61. Wu T, Wang MC, Jing L, Liu ZY, Guo H, Liu Y, Bai YY, Cheng YZ, Nan KJ and Liang X: Autophagy facilitates lung adenocarcinoma resistance to cisplatin treatment by activation of AMPK/mTOR signaling pathway. Drug Des Devel Ther 9: 6421-6431,2015

62. Xu Z, Han X, Ou D, Liu T, Li Z, Jiang G, Liu J and Zhang J: Targeting PI3K/AKT/mTOR-mediated autophagy for tumor therapy. Appl Microbiol Biotechnol 104: 575-587, 2020.

This work is licensed under a Creative Commons Attribution-NonCommercial-NoDerivatives 4.0 International (CC BY-NC-ND 4.0) License. 